\title{
Paradoxal Trends in Azole-Resistant Aspergillus fumigatus in a National Multicenter Surveillance Program, the Netherlands, 2013-2018
}

\author{
Pieter P.A. Lestrade, Jochem B. Buil, Martha T. van der Beek, Ed J. Kuijper, Karin van Dijk, \\ Greetje A. Kampinga, Bart J.A. Rijnders, Alieke G. Vonk, Sabine C. de Greeff, Annelot F. Schoffelen, \\ Jaap van Dissel, Jacques F. Meis, Willem J.G. Melchers, Paul E. Verweij
}

We investigated the prevalence of azole resistance of $A s-$ pergillus fumigatus isolates in the Netherlands by screening clinical $A$. fumigatus isolates for azole resistance during 2013-2018. We analyzed azole-resistant isolates phenotypically by in vitro susceptibility testing and for the presence of resistance mutations in the Cyp51A gene. Over the 6-year period, $508(11 \%)$ of 4,496 culture-positive patients harbored an azole-resistant isolate. Resistance frequency increased from 7.6\% (95\% Cl 5.9\%-9.8\%) in 2013 (58/760 patients) to $14.7 \%(95 \% \mathrm{Cl} 12.3 \%-17.4 \%)$ in 2018 (112/764 patients) $(p=0.0001) . \mathrm{TR}_{34} / \mathrm{L} 98 \mathrm{H}(69 \%)$ and $\mathrm{TR}_{46} /$ Y121F/T289A (17\%) accounted for $86 \%$ of Cyp51A mutations. However, the mean voriconazole $\mathrm{MIC}$ of $\mathrm{TR}_{34} / \mathrm{L} 98 \mathrm{H}$ isolates decreased from $8 \mathrm{mg} / \mathrm{L}$ (2013) to $2 \mathrm{mg} / \mathrm{L}$ (2018), and the voriconazole-resistance frequency was $34 \%$ lower in 2018 than in $2013(p=0.0001)$. Our survey showed changing azole phenotypes in $\mathrm{TR}_{34} / \mathrm{L} 98 \mathrm{H}$ isolates, which hampers the use of current PCR-based resistance tests.

Author affiliations: VieCuri Hospital, Venlo, the Netherlands (P.P.A. Lestrade); Center of Expertise in Mycology Radboud University Medical Center/Canisius-Wilhelmina Hospital, Nijmegen, the Netherlands (J.B. Buil, J.F. Meis, W.J.G. Melchers, P.E. Verweij); Leiden University Medical Center, Leiden, the Netherlands (M.T. van der Beek, E.J. Kuijper); Amsterdam University Medical Center, Vrije Universiteit Amsterdam, Amsterdam, the Netherlands (K. van Dijk); University of Groningen, University Medical Center Groningen, Groningen, the Netherlands (G.A. Kampinga); Erasmus Medical Center, Rotterdam, the Netherlands (B.J.A. Rijnders, A.G. Vonk); Center for Infectious Disease Control, Dutch National Institute for Public Health and the Environment, Bilthoven, the Netherlands (S.C. de Greeff, A.F. Schoffelen, J. van Dissel)

DOI: https://doi.org/10.3201/eid2607.200088
A spergillus fumigatus is a saprobic mold that thrives A on decaying plant material. The fungus is thermotolerant and exhibits optimum growth at $37^{\circ} \mathrm{C}$. A. fumigatus has evolved into an major cause of pulmonary infections, especially in immunocompromised persons. Patients at risk for invasive aspergillosis include patients with hematologic malignancy, solid organ transplant recipients, and patients receiving corticosteroids. In addition, new risk groups are being recognized, including patients treated with ibrutinib (1) and patients with severe influenza $(2,3)$. The fungus might also cause chronic pulmonary infections, chronic lung colonization, and allergic syndromes (4).

Azoles represent the most important class of antifungal agents that are used for the management of Aspergillus diseases. Triazoles with activity against aspergilli include itraconazole, voriconazole, posaconazole, and isavuconazole. However, use of this drug class has been threatened by the emergence of azole resistance, which was first reported in 1997 (5). Although resistance might be selected during azole therapy, resistance selection in the environment through exposure to azole fungicides has been shown to be the most important route for resistance selection (6). The environmental route of resistance selection poses numerous challenges for patient management because two thirds of patients with azole-resistant invasive aspergillosis have no previous history of azole therapy (7). A recent cohort study showed that voriconazole resistance resulted in $21 \%$ lower day-42 survival in patients with culture-positive invasive aspergillosis compared with voriconazole-susceptible infection, indicating a major effect of resistance on patient survival (8). Because most patients with invasive aspergillosis are culture 
negative, sensitive non-culture-based resistance tests are urgently needed (9).

Although azole-resistant $A$. fumigatus was recently added to the antibiotic threats list of the US Centers for Disease Control and Prevention (10), systematic resistance surveillance programs are currently lacking. Surveillance is hampered by low numbers of culture-positive patients, difficulty in diagnosing and classifying patients with Aspergillus diseases, low awareness of fungal resistance, and limited prioritization of fungal resistance research. Furthermore, unlike bacteria, molds do not routinely undergo resistance testing in most clinical microbiology laboratories, thus necessitating the implementation of specific laboratory protocols. Early reports on azole resistance in the Netherlands prompted the national Center for Infectious Disease Control (Cib) to support a reference laboratory to set up a surveillance network to monitor trends in azole-resistance frequency in A. fumigatus. Our aim was to determine the resistance frequency over a period of 6 years to describe resistance phenotypes and to analyze underlying resistance mutations and trends.

\section{Methods}

Five University Medical Centers (UMCs) participated in the surveillance network, including Leiden UMC (Leiden), Erasmus Medical Center (Rotterdam), Amsterdam UMC, VU Medical Center (Amsterdam), UMC Groningen (Groningen), and Radboud UMC (Nijmegen). The geographic regions include the west of the Netherlands (Rotterdam, Leiden, and Amsterdam), which is the most heavily populated region; the north (Groningen); and the east (Nijmegen). The centers were asked to screen A. fumigatus isolates cultured from clinical specimens by using an agarbased screening test (VIPcheck; MediaProducts, https://www.mediaproductsbv.nl). VIPcheck contains 3 agar wells supplemented with itraconazole, voriconazole, and posaconazole, and a growth control well (11). A. fumigatus colonies from the primary culture were inoculated on the 4-wells plate, incubated for up to 48 hours, and inspected for growth. If an isolate grew on any of the azole-containing wells, the isolate was sent anonymously to Radboud UMC for MIC testing and genotypic characterization. MIC testing was performed according to the European Committee on Antimicrobial Susceptibility Testing (EUCAST) broth microdilution reference method (12-14) for amphotericin B (AmB), itraconazole, voriconazole, posaconazole, and isavuconazole (added in 2015 after the drug was clinically licensed). Azole resistance was defined as resistance to $\geq 1$ azole drug, according to EUCAST clinical breakpoints (itraconazole, $>2 \mathrm{mg} / \mathrm{L}$; voriconazole, $>2 \mathrm{mg} / \mathrm{L}$; posaconazole, $>0.25 \mathrm{mg} / \mathrm{L}$, and isavuconazole, $>1 \mathrm{mg} / \mathrm{L})$. EUCAST broth microdilution plates were made at Radboud UMC in batches of 250 96-well plates and complied with the recommended quality control standards (11-13). For A. fumigatus isolates with a confirmed azole-resistant phenotype, the full Cyp51A gene was analyzed by PCR amplification and sequencing (7). The Cyp51A sequence (GenBank accession no. AF338659) was used for mutation analysis. A spore suspension of all isolates was stored at $-80^{\circ} \mathrm{C}$ in $10 \%$ glycerol.

Results of phenotypic testing were sent to the surveillance laboratories as soon as these were available. Analysis of the resistance genotypes was batched, and once a year each center received a list of isolates with resistance genotype and phenotype. The list of isolates was checked by the centers, who also provided the number of $A$. fumigatus culture-positive patients who had been screened for azole resistance during the year and the number of patients who harbored an azole-resistant isolate. Clinical information regarding underlying disease and classification of Aspergillus disease was not collected. Data on A. fumigatus resistance epidemiology are reported and published annually (15).

We calculated mean MICs with 95\% CIs with GraphPad Prism 5.03 (https:/ / www.graphpad.com). For calculations, we recoded MICs $>16 \mathrm{mg} / \mathrm{L}$ as 32 $\mathrm{mg} / \mathrm{L}$. We calculated statistical tests on differences in MIC distributions by using Kruskal-Wallis test and tests on differences in classification according to clinical breakpoints by using the Fisher exact test.

\section{Results}

\section{General Epidemiology}

During 2013-2018, A. fumigatus isolates from 4,518 culture-positive patients were screened for the presence of azole-resistance. In 1 center, prospective screening was not performed in 2015 , but only selected isolates from 22 patients were analyzed (Table 1). Therefore, we excluded these patients from calculation of the azole-resistance frequency, leaving 4,496 patients who had been screened for azole resistance. In total, 508 patients $(11 \%)$ harbored an azole-resistant A. fumigatus isolate. Over the 6-year period, the overall resistance frequency increased from $7.6 \%(95 \% \mathrm{CI}$ $5.9 \%-9.8 \%$ ) in 2013 (58/760 patients) to $14.7 \%$ (95\% CI $12.3 \%-17.4 \%)$ in 2018 (112/764 patients; $\mathrm{p}=0.0001)$.

\section{Triazole-Resistance Genotypes}

Overall, 640 A. fumigatus isolates (obtained from 508 patients) exhibited phenotypical resistance for 
Table 1. Number of Aspergillus fumigatus culture-positive patients screened for azole resistance and azole resistance frequency in clinical $A$. fumigatus isolates in 5 University Medical Centers in the Netherlands*

\begin{tabular}{|c|c|c|c|c|c|c|}
\hline \multirow[b]{2}{*}{ Surveillance center } & \multicolumn{6}{|c|}{ No. resistant/no. screened (\%) } \\
\hline & 2013 & 2014 & 2015 & 2016 & 2017 & 2018 \\
\hline Erasmus MC, Rotterdam & $10 / 231(4.3)$ & $10 / 265(3.8)$ & $7 / 22(31.8) \dagger$ & $24 / 186(12.9)$ & 19/147 (12.9) & $17 / 129(13.2)$ \\
\hline LUMC, Leiden & 19/99 (19.2) & $15 / 113(13.3)$ & $23 / 141(16.3)$ & $18 / 88(20.5)$ & $27 / 114(23.7)$ & $25 / 120(20.8)$ \\
\hline Radboud UMC, Nijmegen & $6 / 123(4.9)$ & $7 / 143(4.9)$ & $12 / 145(8.3)$ & $20 / 210(9.5)$ & $21 / 198(10.6)$ & $23 / 196(11.7)$ \\
\hline UMCG, Groningen & $16 / 194(8.2)$ & $18 / 191(9.4)$ & $15 / 225(6.7)$ & $26 / 215(12.1)$ & $35 / 240(14.6)$ & $34 / 238(14.3)$ \\
\hline VUMC, Amsterdam & $8 / 113(7.1)$ & $9 / 104(8.7)$ & $14 / 89(15.7)$ & $13 / 85(15.3)$ & $12 / 75(16.0)$ & $13 / 81(16.0)$ \\
\hline Total & $58 / 760(7.6)$ & $59 / 814(7.2)$ & $64 / 600(10.7) \ddagger$ & $101 / 784(12.9)$ & $114 / 774(14.7)$ & $112 / 764(14.7)$ \\
\hline
\end{tabular}

$\geq 1$ triazole. $\mathrm{TR}_{34} / \mathrm{L} 98 \mathrm{H}$ was the most frequently observed resistance mechanism and was present in 445 (69\%) azole-resistant $A$. fumigatus isolates, whereas $\mathrm{TR}_{46} / \mathrm{Y} 121 \mathrm{~F} / \mathrm{T} 289 \mathrm{~A}$ was present in 111 (17\%) isolates. Of $445 \mathrm{TR}_{34} / \mathrm{L} 98 \mathrm{H}$ isolates, 24 had $\geq 1$ additional polymorphisms in the Cyp51A gene (F495I, $\mathrm{n}=9$; $\mathrm{Q} 259 \mathrm{H}, \mathrm{n}=5 ; \mathrm{S} 297 \mathrm{~T}, \mathrm{n}=4 ; \mathrm{D} 262 \mathrm{~N}, \mathrm{n}=1 ; \mathrm{N} 326 \mathrm{H}, \mathrm{n}=$ $1 ; \mathrm{P} 337 \mathrm{~L}, \mathrm{n}=1 ; \mathrm{Y} 341 \mathrm{H}, \mathrm{n}=1$; I364V, $\mathrm{n}=1 ; \mathrm{G} 328 \mathrm{~A}, \mathrm{n}$ $=1$; and $\mathrm{L} 399 \mathrm{~V}, \mathrm{n}=1$ ). In addition, $8 \mathrm{TR}_{34} / \mathrm{L} 98 \mathrm{H}$ isolates harbored a T67G substitution in the gene promotor region, which has not been associated with azole resistance. TR-mediated resistance mutations are associated with resistance selection in the environment, which thus accounted for $86 \%$ of resistance mutations. In 76 azole-resistant isolates (12\%), no mutations were found in the Cyp51A gene, indicating that other, yet uncharacterized, resistance mechanisms might be present. Over the 6-year observation period, no significant trends in the distribution of resistance mutations was observed (Figure 1).

\section{Triazole-Resistance Phenotypes}

Resistance mutations most commonly affected the activity of all 4 mold-active azoles. Among the 640 azole-resistant $A$. fumigatus isolates, 413 (65\%) isolates exhibited a panazole-resistant phenotype, 51 (8\%) a multiazole-resistant phenotype, and 176 (28\%) resistance to a single azole.

Because voriconazole is the treatment of choice for invasive aspergillosis, the azole resistance phenotypes were categorized according to voriconazole clinical breakpoints. Overall, 498 (77.8\%) A. fumigatus isolates were voriconazole-resistant (Table 2). Although most voriconazole-resistant isolates exhibited a panazole-resistant phenotype, $50(10 \%)$ voriconazole-resistant isolates were itraconazole-susceptible, of which 8 were also susceptible to posaconazole. Isolated posaconazole susceptibility in voriconazoleresistant isolates was not observed. The underlying resistance mutations detected in these 50 isolates included $\mathrm{TR}_{46} / \mathrm{Y} 121 \mathrm{~F} / \mathrm{T} 289 \mathrm{~A}$ (35 isolates) and G448S (3 isolates), whereas Cyp51A mutations were not detected in 12 isolates. All voriconazole-resistant isolates were also resistant to isavuconazole.

In 124 (19\%) A. fumigatus isolates, a voriconazole MIC of $2 \mathrm{mg} / \mathrm{L}$ was measured (Table 2); of these, $120(97 \%)$ were resistant against either itraconazole, posaconazole, or both. The 4 remaining isolates were

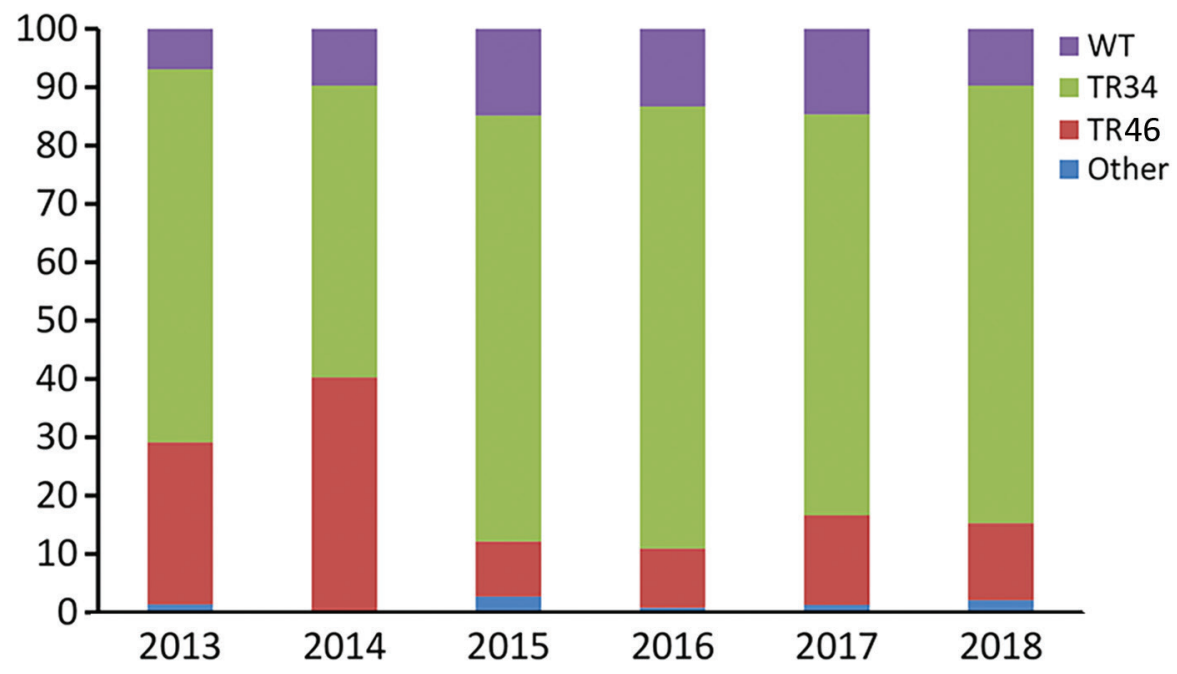

Figure 1. Distribution of Cyp51Amediated resistance mutations in Aspergillus fumigatus, as observed in a national multicenter surveillance program in the Netherlands, 2013-2018. WT, wildtype Cyp51A; TR34, $\mathrm{TR}_{34} / \mathrm{L} 98 \mathrm{H} ; \mathrm{TR} 46, \mathrm{TR}_{46} / \mathrm{Y} 121 \mathrm{~F}$. 
Table 2. Resistance profiles of 640 azole-resistant Aspergillus fumigatus isolates classified according to voriconazole clinical breakpoints in a national multicenter surveillance program in the Netherlands, 2013-2018*

\begin{tabular}{|c|c|c|c|}
\hline \multirow[b]{2}{*}{ Voriconazole classification (no. isolates) } & \multicolumn{3}{|c|}{ No. $(\%)$ isolates } \\
\hline & Itraconazole & Posaconazole & Isavuconazole \\
\hline \multicolumn{4}{|l|}{ Voriconazole-susceptible (18) } \\
\hline Susceptible & 0 & $1(5.6)$ & $6 / 16(37.5)$ \\
\hline Intermediate & $1(5.6)$ & $2(11.1)$ & NA \\
\hline Resistant & $17(94.4)$ & $15(83.3)$ & $10 / 16(62.5)$ \\
\hline \multicolumn{4}{|l|}{ Voriconazole-intermediate (124) } \\
\hline Susceptible & 0 & $4(3.2)$ & $2 / 121(1.7)$ \\
\hline Intermediate & $6(4.8)$ & $19(15.3)$ & NA \\
\hline Resistant & $118(95.2)$ & $101(81.5)$ & $119 / 121(98.3)$ \\
\hline \multicolumn{4}{|l|}{ Voriconazole-resistant (498) } \\
\hline Susceptible & $50(10)$ & $8(1.6)$ & $0 / 396(0)$ \\
\hline Intermediate & $25(5)$ & $28(5.6)$ & NA \\
\hline Resistant & $423(85)$ & $462(92.8)$ & $396 / 396(100)$ \\
\hline
\end{tabular}

itraconazole- and posaconazole-susceptible but isavuconazole-resistant. Three of these isolates harbored the $\mathrm{TR}_{34} / \mathrm{L} 98 \mathrm{H}$ mutation, and in the fourth isolate, no $\mathrm{Cy}$ p51A mutations were found. An underlying resistance mutation was detected in $99(80 \%)$ of the 124 isolates with voriconazole-intermediate susceptibility.

Overall, 18 (3\%) azole-resistant isolates were phenotypically voriconazole-susceptible, although these isolates were resistant to either itraconazole, posaconazole, or both (Table 2). Isavuconazole MICs were available for 16 phenotypically voriconazolesusceptible isolates, and isavuconazole resistance was found in $10(63 \%)$ of these, with a mean isavuconazole MIC of $11 \mathrm{mg} / \mathrm{L}$ (range 2 to $>16 \mathrm{mg} / \mathrm{L}$ ). Underlying Cyp51A-mediated resistance mutations in these 10 isavuconazole-resistant, voriconazole-susceptible isolates included 6 isolates with a $\mathrm{TR}_{34} / \mathrm{L} 98 \mathrm{H}$ genotype, although in 4 isolates no Cyp51A mutations were detected.

$\mathrm{TR}_{34} / \mathrm{L} 98 \mathrm{H}$ is associated with high resistance to itraconazole, which was the case in 438 of 445 (98\%) isolates, although $\mathrm{TR}_{46} / \mathrm{Y} 121 \mathrm{~F} / \mathrm{T} 289 \mathrm{~A}$ is associated with high voriconazole resistance, which was found in all $111 \mathrm{TR}_{46} / \mathrm{Y} 121 \mathrm{~F} / \mathrm{T} 289 \mathrm{~A}$ isolates (Figure 2). Thirty-five of 111 (32\%) TR $\mathrm{TR}_{46} / \mathrm{Y} 121 \mathrm{~F} / \mathrm{T} 289 \mathrm{~A}$ isolates were susceptible to itraconazole, although 3 of these isolates were also susceptible to posaconazole.

\section{Trends in Voriconazole Resistance}

In 2013, voriconazole resistance was detected in $94 \%$ (68/72) of azole-resistant $A$. fumigatus isolates, but in 2018 voriconazole resistance was detected for only $60 \%(87 / 144)$ azole-resistant $A$. fumigatus isolates ( $\mathrm{p}$ $=0.0001$ ) (Figure 3 ). The trend toward lower voriconazole MICs was not attributable to a shift in resistance mutations but was apparent mainly in isolates harboring $\mathrm{TR}_{34} / \mathrm{L} 98 \mathrm{H}$ (Figure 4, panel A). Voriconazole MIC distributions were significantly different when
2013 (mean voriconazole MIC $8 \mathrm{mg} / \mathrm{L}$ ) was compared with 2018 (mean voriconazole MIC $2 \mathrm{mg} / \mathrm{L}$ ) $(\mathrm{p}<0.001)$. In $2013,2(4 \%)$ of $46 \mathrm{TR}_{34} / \mathrm{L} 98 \mathrm{H}$ isolates were not classified as voriconazole-resistant, whereas $49(4 \%)$ of $108(45 \%) \mathrm{TR}_{34} / \mathrm{L} 98 \mathrm{H}$ isolates exhibited a voriconazole-nonresistant phenotype in 2018 ( $p=$ 0.0001), of which most exhibited a MIC of $2 \mathrm{mg} / \mathrm{L}$ (intermediate). Because a F495I mutation in $\mathrm{TR}_{34} / \mathrm{L} 98 \mathrm{H}$ isolates was shown to be associated with reduced resistance to voriconazole (16), all 106 voriconazolenonresistant $\mathrm{TR}_{34} / \mathrm{L} 98 \mathrm{H}$ isolates were checked for the presence of this mutation. Only $8 \mathrm{TR}_{34} / \mathrm{L} 98 \mathrm{H}$ isolates were found to harbor the F495I mutation.

Because MIC testing was performed on receipt of the isolate over a 6-year period and thus involved various batches of MIC plates, all $46 \mathrm{TR}_{34} / \mathrm{L} 98 \mathrm{H}$ isolates from 2013 and 106 isolates (2 isolates were not available) from 2018 were retested for voriconazole and isavuconazole by using a single batch of MIC plates. Retesting confirmed the initial observation showing a $45 \%$ lower voriconazole-resistance frequency among $\mathrm{TR}_{34} / \mathrm{L} 98 \mathrm{H}$ isolates in $2018 \mathrm{com}$ pared with 2013 (46/46 in 2013 vs. 58/106 in 2018; $\mathrm{p}=0.0001$ ) (Appendix Figure, https://wwwnc.cdc. gov/EID/article/26/7/20-0088-App1.pdf). In 2013, the overall azole-resistance frequency was $7.6 \%$ at the patient level and voriconazole-resistance frequency was $7.2 \%$, whereas in 2018 the azole-resistance frequency was $14.7 \%$ compared with $8.8 \%$ voriconazole resistance (Table 1 [estimated voriconazole resistance frequencies not shown]).

We observed a similar decreasing trend of MICs for isavuconazole (Figure 4, panel B; Appendix Figure). However, the decrease in MICs did not result in an increase of susceptible isolates because all but 2 $\mathrm{TR}_{34} / \mathrm{L} 98 \mathrm{H}$ isolates remained isavuconazole-resistant based on the EUCAST breakpoint. The isavuconazole MIC distributions were significantly different when 
the distribution of 2015 was compared with that of $2018(\mathrm{p}<0.001)$. No trends in phenotype changes were observed for itraconazole and posaconazole.

\section{Discussion}

Our resistance surveillance showed an increasing frequency of azole resistance in clinical A. fumigatus isolates during 2013-2018 in the Netherlands. Although the resistance frequency varied between the 5 UMCs, the increasing trend was observed in all centers. In 2017 and 2018, the azole-resistance frequency exceeded $10 \%$ in all centers, a threshold above which experts recommend reconsidering the use of first-line voriconazole monotherapy $(17,18)$. Alternative regimens that cover resistance include voriconazole/isavuconazole in combination with liposomal-AmB or an echinocandin, or monotherapy with liposomal-AmB. As such, the Netherlands national guideline was revised and now recommends combination therapy in patients with suspected invasive aspergillosis, in particular for critically ill patients or when azole resistance cannot be excluded (19).

How the $10 \%$ resistance threshold should be determined remains unclear $(17,18)$. Our study indicated that despite the increasing azole-resistance frequency, the resistance frequency of voriconazole remained below this threshold (8.8\% in 2018). This observation warrants the question of which threshold (i.e., azole resistance or voriconazole resistance) should be used to guide decisions regarding primary treatment choices. The trend toward lower voriconazole MICs was observed mainly in $\mathrm{TR}_{34} / \mathrm{L} 98 \mathrm{H}$ isolates, whereas most nonresistant isolates exhibited a voriconazole MIC of $2 \mathrm{mg} / \mathrm{L}$ (intermediate). The optimal management of voriconazole-intermediate invasive aspergillosis remains unclear, but an increased failure rate is anticipated in patients treated with voriconazole monotherapy at the standard dose. An increased voriconazole trough level target of 2-6 $\mathrm{mg} / \mathrm{L}$ is recommended or combining voriconazole with an echinocandin or liposomal-AmB monotherapy (18). Furthermore, there is currently insufficient evidence that infection caused by azole-susceptible or intermediate susceptible $A$. fumigatus isolates that harbor a resistance mutation can safely be treated with azole monotherapy.

Resistance was highly dominated by environmental resistance mutations, such as $\mathrm{TR}_{34} / \mathrm{L} 98 \mathrm{H}$ and $\mathrm{TR}_{46} / \mathrm{Y} 121 \mathrm{~F} / \mathrm{T} 289 \mathrm{~A}$. Indeed, a recent study identified sites at high risk for resistance selection in the environment, referred to as hotspots (20). Particularly, stockpiling of decaying plant waste containing azole fungicide residues was found to harbor high numbers of resistant $A$. fumigatus, and isolates with identical resistance mutations to those recovered from clinical specimens were cultured from these hotspots $(20,21)$. The observations support a strong link between environmental resistance selection and azole-resistant disease in humans. The increasing trend in azole-resistance frequency and the emergence of new resistance mutations indicate that the current use of azole fungicides is not sustainable and over time the medical use of azoles will be further
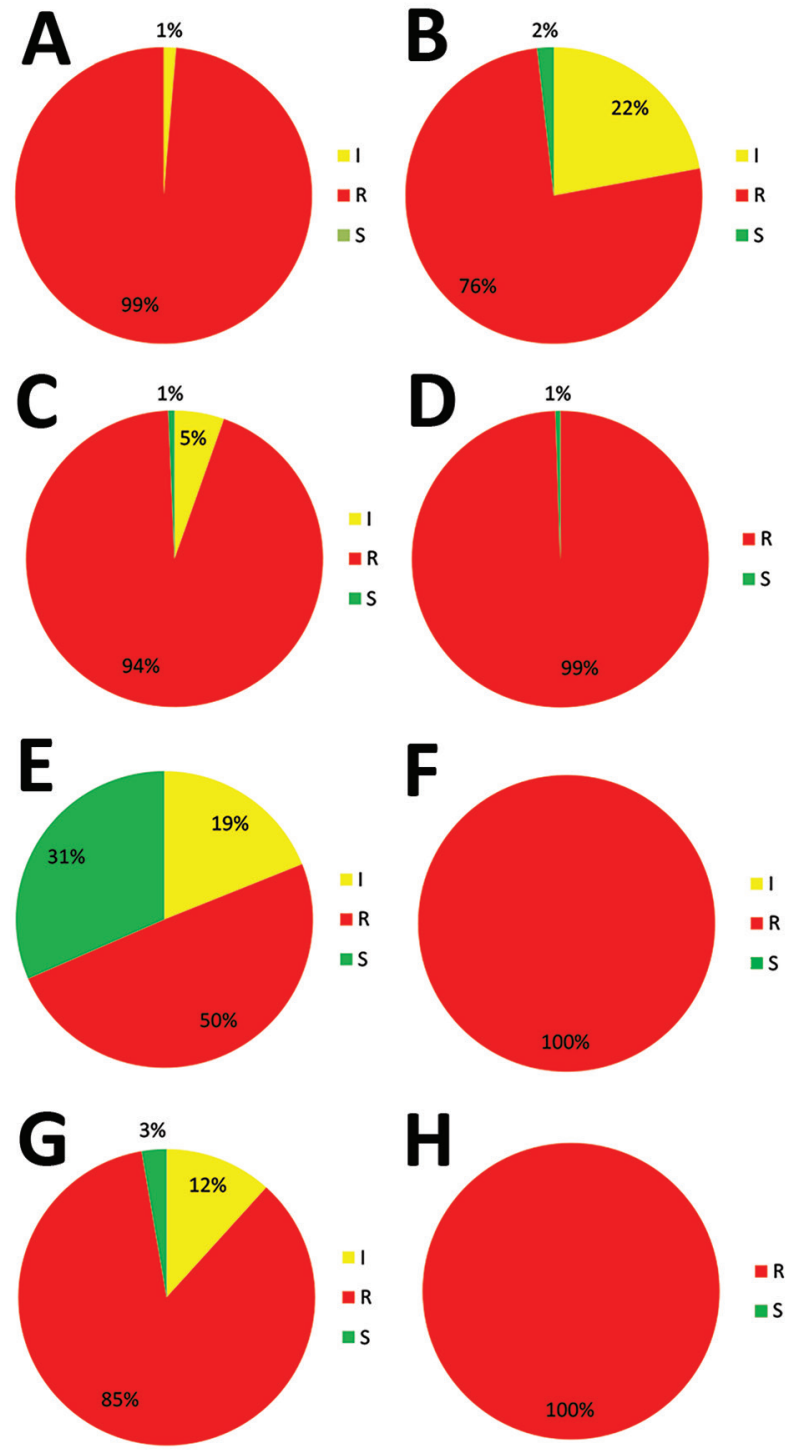

Figure 2. Triazole-resistance classification in 555 Aspergillus fumigatus isolates harboring $\mathrm{TR}_{34} / \mathrm{L} 98 \mathrm{H}$ and $\mathrm{TR}_{46} / \mathrm{Y} 121 \mathrm{~F} / \mathrm{T} 289 \mathrm{~A}$ resistance mutations, as observed in a national multicenter surveillance program in the Netherlands, 2013-2018. A) Itraconazole $\mathrm{TR}_{34} / \mathrm{L} 98 \mathrm{H}$. B) Voriconazole $\mathrm{TR}_{34} / \mathrm{L} 98 \mathrm{H}$. C) Posaconazole $\mathrm{TR}_{34} / \mathrm{L} 98 \mathrm{H}$. D) Isavuconazole $\mathrm{TR}_{34} / \mathrm{L} 98 \mathrm{H}$. E) Itraconazole $\mathrm{TR}_{46} / \mathrm{Y} 121 \mathrm{~F} / \mathrm{T} 289 \mathrm{~A}$. F) Voriconazole, $\mathrm{TR}_{46} / \mathrm{Y} 121 \mathrm{~F} /$ T289A. G) Posaconazole $\left.T_{46} / Y 121 F / T 289 A . H\right)$ Isavuconazole $\mathrm{TR}_{46} / \mathrm{Y} 121 \mathrm{~F} / \mathrm{T} 289 \mathrm{~A}$. I, intermediate; $\mathrm{R}$, resistant; S, susceptible. 


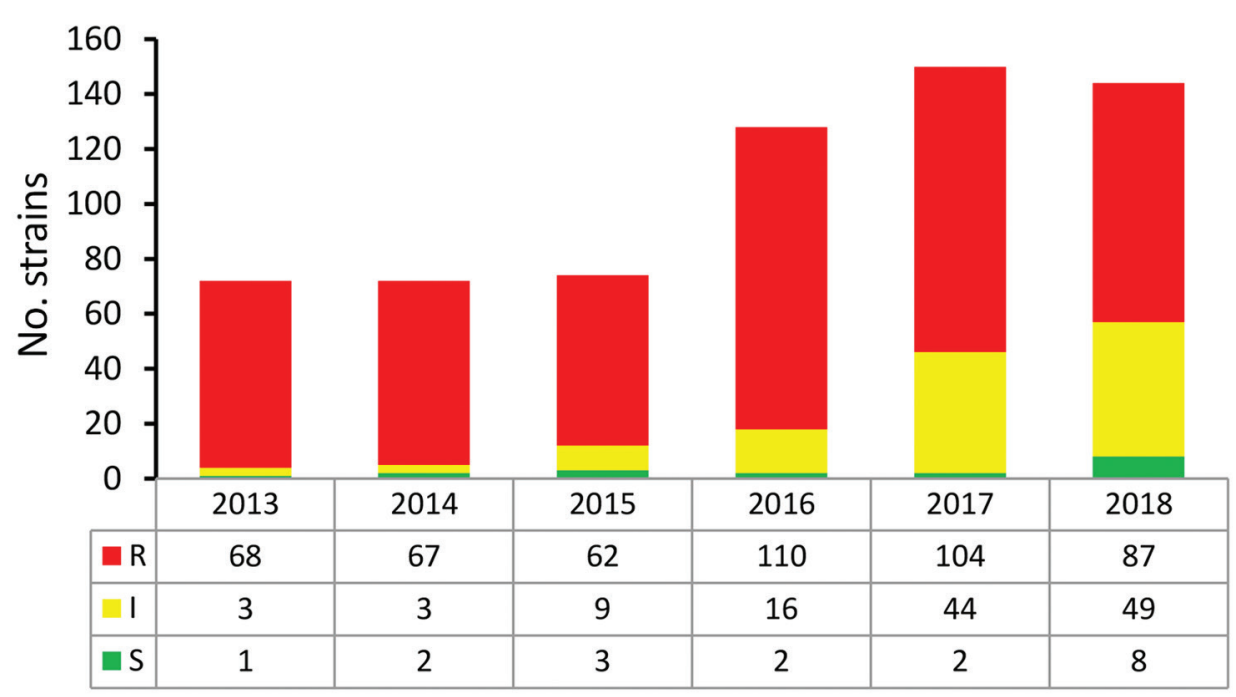

Figure 3. Trends in voriconazolesusceptibility classification of 640 Aspergillus fumigatus isolates, by year, as observed in a national multicenter surveillance program in the Netherlands, 2013-2018. I, intermediate; R, resistant; S, susceptible.

threatened. Given the limited alternative treatment options for Aspergillus diseases, further research aimed at reducing the burden of azole resistance in the environment is urgently needed.

An important question is how the changing voriconazole phenotype in $\mathrm{TR}_{34} / \mathrm{L} 98 \mathrm{H}$ isolates can be explained. One possibility is that $\mathrm{TR}_{34} / \mathrm{L} 98 \mathrm{H}$ isolates harbor additional mechanisms or compensatory mutations that affect the overall azole-resistance phenotype. Additional changes in peptide sequence of the 14-a-sterol demethylase enzyme have been reported in $\mathrm{TR}_{34} / \mathrm{L} 98 \mathrm{H}$ isolates, such as $\mathrm{F} 495 \mathrm{I}$, which confers resistance to imidazole (16). Recombination experiments showed that recombinants with S297T/ $\mathrm{F} 495 \mathrm{I}$ in the $\mathrm{TR}_{34} / \mathrm{L} 98 \mathrm{H}$ background conferred high resistance to imidazole but also produced lower voriconazole MICs compared with the $\mathrm{TR}_{34} / \mathrm{L} 98 \mathrm{H}$ parent strain (16). Because $\mathrm{TR}_{34}$ isolates originate in the environment through exposure to azole fungicides, changes in azole fungicide exposures could prompt changes in resistance phenotypes. However, only 9 isolates with an additional F495I mutation in the Cyp51A gene were found, of which 8 were voriconazole-susceptible, indicating that other or multiple factors might have contributed to the observed phenotype change.

Another possibility is the presence of other resistance mechanisms in $\mathrm{TR}_{34} / \mathrm{L} 98 \mathrm{H}$ isolates. Recent studies analyzing transcriptional control mechanisms of Cyp51A, have identified transcription factors, such as $A B C$ transporter regulator, which was found to regulate many different processes involved in drug resistance, metabolism, and virulence (22). Furthermore, other steps in the ergosterol biosynthesis might be affected, for example, through mutations in the 3-hydroxy-3-methyl-glutaryl-coenzyme A reductaseencoding gene (hmg1) (23). Mutations in the $h m g 1$ gene resulting in peptide sequence changes were found to be frequent in $\mathrm{TR}_{34} / \mathrm{L} 98 \mathrm{H}$ isolates, possibly affecting the azole phenotype (23). These recent insights indicate that the resistance phenotype is likely to be multifactorial and that more research is needed to characterize possible mechanisms that explain the observed variation in $\mathrm{TR}_{34} / \mathrm{L} 98 \mathrm{H}$ phenotypes.

The main clinical implication of the observed phenotypic variation in $\mathrm{TR}_{34} / \mathrm{L} 98 \mathrm{H}$ relates to direct detection of resistance mutations through PCR tests. Several commercial PCR tests are available that enable detection of $\mathrm{TR}_{34} / \mathrm{L} 98 \mathrm{H}$ and $\mathrm{TR}_{46} / \mathrm{Y} 121 \mathrm{~F} / \mathrm{T} 289 \mathrm{~A}$ directly in clinical specimens $(24,25)$, which are used to guide selection of antifungal drugs. However, this approach can only be used if the resistance genotype predicts the azole phenotype. Although $\mathrm{TR}_{46} / \mathrm{Y} 121 \mathrm{~F} /$ T289A mutation detection is uniformly associated with resistance to voriconazole, the wide spectrum of voriconazole MICs in $\mathrm{TR}_{34} / \mathrm{L} 98 \mathrm{H}$ isolates hampers the use of direct detection of this mutation because voriconazole therapy might be withheld in cases of voriconazole-susceptible infection. However, as stated previously, the efficacy of voriconazole in voriconazole-susceptible and voriconazole-intermediate $\mathrm{TR}_{34} / \mathrm{L} 98 \mathrm{H}$ infection would need to be investigated before any treatment recommendations involving azole monotherapy can be implemented.

Our resistance surveillance had several limitations. All A. fumigatus isolates cultured from clinical specimens were screened for azole resistance, involving diverse patient groups and isolates not regarded clinically relevant. Including all isolates has the advantage of collecting a meaningful number 
of isolates, but the resistance frequency might not be representative for specific patient groups such as those with invasive aspergillosis. Furthermore, within hospitals the resistance frequency might vary between years (26), thus further complicating establishing a meaningful (local) resistance frequency. Another limitation was the lack of clinical information and Aspergillus disease classification. Such information would be more suitable to guide treatment decisions and local antifungal guidelines, but gathering the data is laborious, given that all aspergillosis cases need to be identified and classified. Various cohort studies have been performed in the Netherlands showing differences in resistance rates between patient groups. A resistance frequency of $26 \%$ (10 of 38 patients) was reported in A. fumigatus culture-positive intensive-care unit patients in a single hospital (27) and 29\% (4 of 14 patients) in a national study of intensive-care unit patients with influenza-associated aspergillosis (2). In contrast, a low resistance frequency was reported in a 5 year single-center cohort of patients with hematologic malignancy (28), but the culture-positivity rate was only $6 \%$ and thus, in most patients, the presence of resistance remained unknown. To date, only few studies have determined the frequency of resistance mutations in culture-negative patients. In 1 study, the rate was found to be similar to that observed in culture-positive patients (29), although higher resistance rates were reported in culture-negative patients in comparison with the rates found by culture in another study on patients with chronic rather than acute invasive aspergillosis (30). Differences could be explained in part by coincidence because the number of cases in these single-center studies is small. Another explanation might be the number of A. fumigatus colonies that were tested for resistance, given that clinical cultures might contain azolesusceptible and azole-resistant colonies in cases of mixed infection $(31,32)$.

In conclusion, azole resistance in A. fumigatus has been reported worldwide and provides major

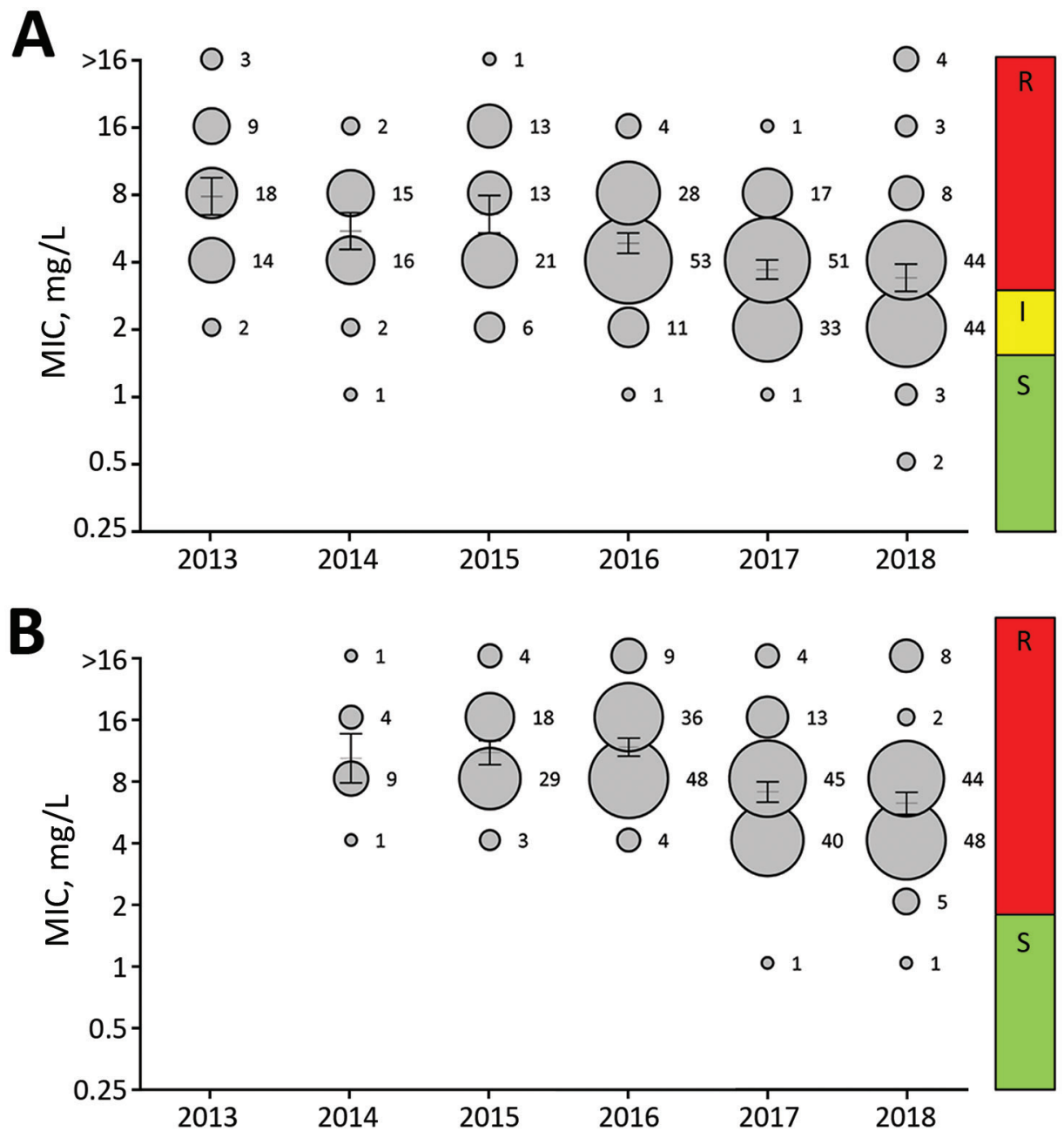

Figure 4. Trends in voriconazole (A) and isavuconazole (B) MIC distributions in Aspergillus fumigatus harboring $\mathrm{TR}_{34}$ I $\mathrm{L} 98 \mathrm{H}$, as observed in a national multicenter surveillance program in the Netherlands, 2013-2018. MIC distribution is displayed as a bubble graph for each year, where the diameter corresponds with the number of isolates with the corresponding MIC. The number of isolates is presented for each MIC. Mean MIC with 95\% Cls are plotted for each year as a line with error bars. The clinical interpretation is shown on the right of the diagram. I, intermediate; R, resistant; $\mathrm{S}$, susceptible. 
challenges regarding management of invasive aspergillosis and other Aspergillus diseases (9,32). Nevertheless, A. fumigatus is not included in global action plans to combat antimicrobial resistance (33), and no international surveillance programs monitor resistance in A. fumigatus. As a consequence, the presence and frequency of azole resistance remains unknown in most countries $(34,35)$. Despite the challenges we face in performing resistance surveillance in A. fumigatus, our national surveillance has proved important to guide the national treatment guideline and provided insights in trends in resistance genotypes and phenotypes. Furthermore, continued surveillance will help to monitor effects of interventions aimed at reducing the resistance burden in the environment. We believe that global A. fumigatus resistance surveillance programs are warranted and should be implemented in initiatives to combat antimicrobial resistance.

\section{Acknowledgments}

We thank our mycology technicians for their dedicated work.

\section{About the Author}

Mr. Lestrade currently works in VieCuri Medical Centre as a clinical microbiologist. His primary research interest is triazole resistance in Aspergillus fumigatus.

\section{References}

1. Ghez D, Calleja A, Protin C, Baron M, Ledoux MP, Damaj G, et al.; French Innovative Leukemia Organization (FILO) CLL group. Early-onset invasive aspergillosis and other fungal infections in patients treated with ibrutinib. Blood. 2018; 131:1955-9. https://doi.org/10.1182/ blood-2017-11-818286

2. van de Veerdonk FL, Kolwijck E, Lestrade PPA, Hodiamont CJ, Rijnders BJA, van Paassen J, et al.; Dutch Mycoses Study Group. Influenza-associated aspergillosis in critically ill patients. Am J Respir Crit Care Med. 2017;196:524-7. https://doi.org/10.1164/rccm.201612-2540LE

3. Schauwvlieghe AFAD, Rijnders BJA, Philips N, Verwijs R, Vanderbeke L, Van Tienen C, et al.; Dutch-Belgian Mycosis Study Group. Invasive aspergillosis in patients admitted to the intensive care unit with severe influenza: a retrospective cohort study. Lancet Respir Med. 2018;6:782-92. https://doi.org/10.1016/S2213-2600(18)30274-1

4. Kosmidis C, Denning DW. The clinical spectrum of pulmonary aspergillosis. Thorax. 2015;70:270-7. https:/ / doi.org/10.1136/thoraxjnl-2014-206291

5. Verweij PE, Chowdhary A, Melchers WJG, Meis JF. Azole resistance in Aspergillus fumigatus: can we retain the clinical use of mold-active antifungal azoles? Clin Infect Dis. 2016;62:362-8. https://doi.org/10.1093/cid/civ885

6. Snelders E, van der Lee HAL, Kuijpers J, Rijs AJMM, Varga J, Samson RA, et al. Emergence of azole resistance in Aspergillus fumigatus and spread of a single resistance mechanism. PLoS Med. 2008;5:e219. https:/ / doi.org/ 10.1371/journal.pmed.0050219

7. van der Linden JWM, Snelders E, Kampinga GA, Rijnders BJA, Mattsson E, Debets-Ossenkopp YJ, et al. Clinical implications of azole resistance in Aspergillus fumigatus, the Netherlands, 2007-2009. Emerg Infect Dis. 2011;17:1846-54. https:// doi.org/10.3201/eid1710.110226

8. Lestrade PPA, Bentvelsen RG, Schauwvlieghe AFAD, Schalekamp S, van der Velden WJFM, Kuiper EJ, et al. Voriconazole resistance and mortality in invasive aspergillosis: a multicenter retrospective cohort study. Clin Infect Dis. 2019;68:1463-71. https:/ / doi.org/10.1093/ cid/ciy859

9. Buil JB, Zoll J, Verweij PE, Melchers WJG. Molecular detection of azole-resistant Aspergillus fumigatus in clinical samples. Front Microbiol. 2018;9:515. https:/ / doi.org/ 10.3389/fmicb.2018.00515

10. US Centers for Disease Control and Prevention. Antibiotic resistance threats in the United States 2019 [cited 2019 Nov 29]. https://www.cdc.gov/drugresistance/pdf/ threats-report/ 2019-ar-threats-report-508.pdf

11. Arendrup MC, Verweij PE, Mouton JW, Lagrou K, Meletiadis J. Multicentre validation of 4-well azole agar plates as a screening method for detection of clinically relevant azole-resistant Aspergillus fumigatus. J Antimicrob Chemother. 2017;72:3325-33. https://doi.org/10.1093/jac/dkx319

12. Hope WW, Cuenca-Estrella M, Lass-Flörl C, Arendrup MC; European Committee on Antimicrobial Susceptibility Testing Subcommittee on Antifungal Susceptibility Testing. EUCAST technical note on voriconazole and Aspergillus spp. Clin Microbiol Infect. 2013;19:E278-80. https://doi.org/10.1111/1469-0691.12148

13. Arendrup MC, Meletiadis J, Mouton JW, Guinea J, Cuenca-Estrella M, Lagrou K, et al.; Subcommittee on Antifungal Susceptibility Testing of the ESCMID European Committee for Antimicrobial Susceptibility Testing. EUCAST technical note on isavuconazole breakpoints for Aspergillus, itraconazole breakpoints for Candida and updates for the antifungal susceptibility testing method documents. Clin Microbiol Infect. 2016;22:571.e1-4. https:/ / doi.org/ 10.1016/j.cmi.2016.01.017

14. Arendrup MC, Cuenca-Estrella M, Lass-Flörl C, Hope WW; European Committee on Antimicrobial Susceptibility Testing Subcommittee on Antifungal Susceptibility Testing. EUCAST technical note on Aspergillus and amphotericin B, itraconazole, and posaconazole. Clin Microbiol Infect. 2012; 18:E248-50. https://doi.org/10.1111/j.1469-0691.2012.03890.x

15. de Greeff SC, Mouton JW, Schoffelen AF, Verduin CM. Nethmap 2019: consumption of antimicrobial agents and antimicrobial resistance among medically important bacteria in the Netherlands [cited 2019 Nov 29]. https:/ / www.rivm. $\mathrm{nl}$ /publicaties/nethmap-2019-consumption-ofantimicrobial-agents-and-antimicrobial-resistance-among

16. Chen Y, Li Z, Han X, Tian S, Zhao J, Chen F, et al. Elevated MIC values of imidazole drugs against Aspergillus fumigatus isolates with $\mathrm{TR}_{34} / \mathrm{L} 98 \mathrm{H} / \mathrm{S} 297 \mathrm{~T} / \mathrm{F} 495 \mathrm{I}$ mutation. Antimicrob Agents Chemother. 2018;62:1-10. https:/ / doi.org/10.1128/ AAC.01549-17

17. Verweij PE, Ananda-Rajah M, Andes D, Arendrup MC, Brüggemann RJ, Chowdhary A, et al. International expert opinion on the management of infection caused by azoleresistant Aspergillus fumigatus. Drug Resist Updat. 2015;2122:30-40. https:// doi.org/10.1016/j.drup.2015.08.001

18. Ullmann AJ, Aguado JM, Arikan-Akdagli S, Denning DW, Groll AH, Lagrou K, et al. Diagnosis and management of Aspergillus diseases: executive summary of the 2017 
ESCMID-ECMM-ERS guideline. Clin Microbiol Infect. 2018; 24(Suppl 1):e1-38. https:/ / doi.org/10.1016/j.cmi.2018.01.002

19. Stichting Werkgroep AntibioticaBeleid. SWAB guidelines for the management of invasive fungal infections [cited 2019 Nov 29]. https://swab.nl/richtlijnen

20. Schoustra SE, Debets AJM, Rijs AJMM, Zhang J, Snelders E, Leendertse PC, et al. Environmental hotspots for azole resistance selection of Aspergillus fumigatus, the Netherlands. Emerg Infect Dis. 2019;25:1347-53. https:// doi.org/10.3201/ eid2507.181625

21. Zhang J, Snelders E, Zwaan BJ, Schoustra SE, Meis JF, van Dijk K, et al. A novel environmental azole resistance mutation in Aspergillus fumigatus and a possible role of sexual reproduction in its emergence. MBio. 2017;8:e00791-17. https:// doi.org/10.1128/mBio.00791-17

22. Paul S, Stamnes M, Thomas GH, Liu H, Hagiwara D, Gomi K, et al. AtrR is an essential determinant of azole resistance in Aspergillus fumigatus. MBio. 2019;10:1-19. https://doi.org/10.1128/mBio.02563-18

23. Rybak JM, Ge W, Wiederhold NP, Parker JE, Kelly SL, Rogers PD, et al. Mutations in $h m g 1$, challenging the paradigm of clinical triazole resistance in Aspergillus fumigatus. MBio. 2019;10:1-19. https:// doi.org/10.1128/mBio.00437-19

24. Dannaoui E, Gabriel F, Gaboyard M, Lagardere G, Audebert L, Quesne G, et al. Molecular diagnosis of invasive aspergillosis and detection of azole resistance by a newly commercialized PCR kit. J Clin Microbiol. 2017;55:3210-8. https:/ / doi.org/10.1128/JCM.01032-17

25. Chong GM, van der Beek MT, von dem Borne PA, Boelens J, Steel E, Kampinga GA, et al. PCR-based detection of Aspergillus fumigatus Cyp51A mutations on bronchoalveolar lavage: a multicentre validation of the AsperGenius assay® in 201 patients with haematological disease suspected for invasive aspergillosis. J Antimicrob Chemother. 2016;71:3528-35. https://doi.org/10.1093/jac/dkw323

26. Buil JB, Snelders E, Denardi LB, Melchers WJG, Verweij PE. Trends in azole resistance in Aspergillus fumigatus, the Netherlands, 1994-2016. Emerg Infect Dis. 2019;25:176-8. https://doi.org/10.3201/eid2501.171925

27. van Paassen J, Russcher A, In 't Veld-van Wingerden AW, Verweij PE, Kuijper EJ. Emerging aspergillosis by azole-resistant Aspergillus fumigatus at an intensive care unit in the Netherlands, 2010 to 2013. Euro Surveill. 2016;21:30300. https://doi.org/10.2807/1560-7917. ES.2016.21.30.30300
28. Lestrade PP, van der Velden WJFM, Bouwman F, Stoop FJ, Blijlevens NMA, Melchers WJG, et al. Epidemiology of invasive aspergillosis and triazole-resistant Aspergillus fumigatus in patients with haematological malignancies: a singlecentre retrospective cohort study. J Antimicrob Chemother. 2018;73:1389-94. https://doi.org/10.1093/jac/dkx527

29. Montesinos I, Argudín MA, Hites M, Ahajjam F, Dodémont M, Dagyaran C, et al. Culture-based methods and molecular tools for azole-resistant Aspergillus fumigatus detection in a Belgian university hospital. J Clin Microbiol. 2017;55:2391-9. https:// doi.org/10.1128/JCM.00520-17

30. Denning DW, Park S, Lass-Florl C, Fraczek MG, Kirwan M, Gore R, et al. High-frequency triazole resistance found in nonculturable Aspergillus fumigatus from lungs of patients with chronic fungal disease. Clin Infect Dis. 2011;52:1123-9. https:/ / doi.org/10.1093/cid/cir179

31. Ahmad S, Joseph L, Hagen F, Meis JF, Khan Z. Concomitant occurrence of itraconazole-resistant and -susceptible strains of Aspergillus fumigatus in routine cultures. J Antimicrob Chemother. 2015;70:412-5. https://doi.org/10.1093/jac/ dku410

32. Kolwijck E, van der Hoeven H, de Sévaux RGL, ten Oever J, Rijstenberg LL, van der Lee HAL, et al. Voriconazolesusceptible and voriconazole-resistant Aspergillus fumigatus coinfection. Am J Respir Crit Care Med. 2016;193:927-9. https:/ / doi.org/10.1164/rccm.201510-2104LE

33. World Health Organization. Global action plan on antimicrobial resistance [cited 2019 Nov 29]. https:/ / www. who.int/antimicrobial-resistance/global-action-plan

34. van der Linden JWM, Arendrup MC, Warris A, Lagrou K, Pelloux H, Hauser PM, et al. Prospective multicenter international surveillance of azole resistance in Aspergillus fumigatus. Emerg Infect Dis. 2015;21:1041-4. https:/ / doi.org/ 10.3201/eid2106.140717

35. Lestrade PPA, Meis JF, Melchers WJG, Verweij PE. Triazole resistance in Aspergillus fumigatus: recent insights and challenges for patient management. Clin Microbiol Infect. 2019;25:799-806. https:// doi.org/10.1016/j. cmi.2018.11.027

Address for correspondence: Paul E. Verweij, Medical Microbiology, Internal postal code: 777, Geert Grooteplein 10, 6525 GA Nijmegen, the Netherlands; email: paul.verweij@radboudumc.nl 